\title{
Reinhard Hoffmann Beamtenstreik und Verfassungsverständnis vom Sozialstaat
}

Im Jahre 1970 ist der Beamtenstreik zu einem immerhin anerkannten Problem und Diskussionsgegenstand für die Rechtswissenschaft geworden, in einem seit Jahrzehnten ungewohnten Austausch der Argumente pro und contra. Dazu trug wesentlich bei die - wenigstens prinzipielle - Bejahung des Beamtenstreikrechts durch die von den Gewerkschaften in Auftrag gegebenen Gutachten von Däubler ${ }^{1}$ und Ramm$^{2}$, welch letzteres in engem Zusammenhang mit der zu ähnlichen Ergebnissen gelangenden Dissertation von Benz ${ }^{\mathbf{3}}$ zu sehen ist.

Diese erhebliche Sinneswandlung der Rechtswissenschaft, die bis dahin sich in der Regel auf den Hinweis auf ein traditionelles Streikverbot beschränkte und abweichende Meinungen nicht einmal notierte, ist keine bloß wissenschaftsimmanente Entwicklung und auch nicht nur auf das Heranwachsen einer neuen Juristengeneration, schon gar nicht von »kritischen« Juristen zurückzuführen. Vielmehr drücken sich darin die praktische Entwicklung der gesellschaftlichen Wirklichkeit im öffentlichen Dienst gegen Ende der 6oer Jahre, die Änderungen in gesellschaftlicher Praxis und Bewußtsein vor allem der Beamten aus, die ihrerseits erst die Gutachtenaufträge der Gewerkschaftsspitzen veranlaßten. 1970 erfolgte der Durchbruch zum Beamtenstreik,$_{5}^{5}$ im Bereich der Hochschulen mit Streiks der Assistenten und wissenschaftlichen Mitarbeiter in Marburg, Bochum und Münster ${ }^{8}$.

Die Gewerkschaft Erziehung und Wissenschaft will einen Streikfonds bilden? Aber es läuft hier keine einsinnig-geradlinige Entwicklung $a b$, sondern eine komplexe Bewußtseins- und Verhaltensänderung einer größeren Anzahl von Menschen, mit häufigen Seitensprüngen und Schwankungen, die Ausdruck einer erheblichen Unsicherheit der Betroffenen sind. Das führt zu mancherlei Ausweichmanövern, so wenn die hessischen Lehrer ihre Proteststreiks während der Unterrichtszeit krampfhaft als nur befristete Protestversammlungen vom Streik abzugrenzen versuchen ${ }^{8}$, die Polizeibeamten einen halbstündigen Streik als

1 Der Streik im öffentlichen Dienst, I 970 - besprochen von Rehbinder DVBl. r970, S. 709, Löwisch DOV 1970, S. 686, Schick JZ 1970, S. 742.

2 Das Koalitions- und Streikrecht der Beamten, I970 - hesprochen von Rehbinder DVBl. I97I, S. 124 .

3 Beamtenverhältnis und Arbeitsverhältnis, 1969 - besprochen von Ule DVBl. I970, S. 709.

4 Wie z. B. noch Maunz in der September 1970 erschienenen Lieferung von Maunz-Dürig-Herzog, GG, Art. 9, Rn. $11_{4}$ Anm. 7, 134 .

s So Ramm S. ro8, ro6 ff. (zur tatsächlichen Entwidklung); dazu auch Däubler, S. 4 f.

- Welt vom 6. 5. 70 und Frankfurter Allgemeine* vom 17.12.70 sowie vom 23. 12.70 mi einem Bericht über den diesbezüglichen Vorwurf des Hochschulverbandes an den hess. Ministerpräsidenten, weinen wilden Beamtenstreik zu politischen Zwecken durch die Fortzahlung der Gehälter finanziert «u haben.

7 .Frankfurter Rundschau vom 23.10. 70 und schon früher vom 6. 5. 69.

8 .Frankfurter Rundschau vom 6. 5. 69. 
*Denkpause* kaschieren ${ }^{9}$ und die Steuerbeamten auf den Dienst nach Vorschrift ${ }^{10}$ ausweichen, den sie zum $*$ Dienst nach Recht und Gesetz $*$ aufwerten ${ }^{11}$. Auf der anderen Seite sprachen sich selbst im Rahmen des Deutschen Beamtenbundes schon 1968 neun von zehn Steuerbeamten für den Streik aus, wenn er rechtlich zulässig ist ${ }^{12}$; ebenso bejahten $93 \%$ von rund 70000 Polizeibeamten eine gleiche Frage der Gewerkschaft der Polizei ${ }^{13}$.

Die Analyse der gesellschaftlichen Bedingungen und Faktoren dieser Bewußtseinswandlung könnte einiges beitragen auch für die rechtliche Einordnung des Streikproblems. Da bislang gründliche sozialwissenschaftliche Untersuchungen über das gesellschaftliche Denken der Beamten, vergleichbar denen über die Arbeiter und die Angestellten, nicht vorliegen, können hier nur allgemeine Beobachtungen über die Struktur des öffentlichen Dienstes und den Beamtenstatus angeführt werden. Das aus der Monarchie und der Weimarer Republik überkommene Berufsbeamtentum war in seiner Beschränkung auf den hoheitlichen Gesetzesvollzug und in seiner Verbindung mit einer absolut gesetzten Staatsidee konzipiert für eine im wesentlichen statische, noch nicht ausschließlich bürgerlichkapitalistische Gesellschaft. Dem daraus resultierenden Standesbewußtsein hatten bereits der soziale Wandel und die grundsätzliche Veränderung der Verfassungsordnung nach 1945 die Basis entzogen. In der Abwehr von Angriffen gegen das in seinem tradierten Bestand 1949 fortgeführte Berufsbeamtentum

- Frankfurter Allgemeine vom 16. 10. 70.

10 Die rechtliche Problematik des Dienstes nach Vorschrift ist auch in den neuen Außerungen noch nicht richtig erfaßt worden, weil dessen soziale Bedingungen nicht erkannt werden. Däubler (S. $258 \mathrm{ff}$.) hat zu Recht das bewußte Mißverstehen von Vorschriften als Schlechterfüllung der Dienstpflichten aus dern Dienst nach Vorschrift ausgeklammert, der im wesentlichen eindeutige Vorshriften ohne Auslegungsspielraum betriff. Diese Vorshriften sind in der Regel nicht individuell fehlerhaft in dem Sinne, daß sie widersprüchlich, zwedswidrig, sinnüberholt * wären, wie es die meisten Autoren aber annehmen; vgl. Ramm, S. 126; auch Däubler, S. 260; vor allem Isensee, Dienst nach Vorschrift als vorschriftswidriger Dienst, JZ 1971, S. 73 ff. - das Manuskript wurde mir dankenswerterweise schon vor Veröffentlichung überlassen -. Der Fehler liegt vielmehr in der hierarchischen Organisation des offentlichen Dienstes mit der mehr oder weniger starren Trennung von Leitung und Ausführung, derzufolge die von oben erlassenen Vorschriften das ausführende Handeln unten in seiner praktischen Komplexität und Spontaneität gar nicht einfangen können. Es handelt sich um das infolge der Hierarchie zwangsläufige Paradoxon $\star$, da $B$ »die reguläre Wirksamkeit der Verwaltung nur durch illegale Maßnahmen der Organwalter aufrecht erhalten wird (Isensee), was Däubler (S. 259 ) am Beispiel des Rangierdienstes der Bundesbahn deutlich macht. Dieser Realität des Verwaltungshandelns steht Isensee hilflos gegenüber und erklärt es für äußerst unwahrscheinlid, daß der Standard eines ganzen Verwaltungszweiges nur durch überobligatorischen Einsatz aufrecht erhalten wird*. Ohne den Sachverhalt zu erkennen, erklärt Isensee den Dienst nach Vorschrift wegen Verstoßes gegen die Verfassung für schlechthin rechtswidrig, indem er die Funktionsfähigkeit und Effizienz der Verwaltung zum Bestandteil der Dienstpflicht macht, jedoch ohne damit eine Verletzung des Normbefehls zulassen zu wollen. Derart geht er an dem eigentlichen Problem vorbei, oder er verwickelt sich in Widersprüche, wenn er ein - Wechselspiel von Spontaneität und Disziplin fordert, das gegenüber eindeutigen Vorschriften eben nicht weiter helfen kann, und wenn er die Beachtung einer Vorschrift in Abweichung von einer Verwaltungsübung, die unter eindeutigem Verstoß gegen die Vorschrift und damit gegen die Hierarchie in der horizontalen Kooperation an der Basis, durch freiwillig übernommene Zusatzleistung entstanden ist und von der Hierarchie offiziell gar nicht akzeptiert werden darf (z. B. bei Sicherheitsvorschriften), als unzulässigen Verstoß gegen diese Hierarchie ansieht. $\mathrm{Da}$ die Hierarchie zwangsläufig an den Vorschriften festhalten muß zur Definition der dienst-, haftungs- und strafrechtlichen Verantwortung der Bediensteten, tragen die letzteren bei Abweichung das Risiko; vgl. Bericht in der Frankfurter Allgemeinen vom 30. I2.68 über die Bestrafung eines Postschaffners, der unter Verstoß gegen die Vorschriften die Paketzustellung vereinfacht hatte, ohne den Eingang der Paketkarten abzuwarten. Deswegen kann der Dienst nach Vorschrift nicht rechtswidrig sein.

11 - Frankfurter Allgemeinea vom I6. 12.70.

12 . Welt a vom I4. 5.68 sowie vom 7.7.70.

13 Heyn, Dürfen, können, müssen Polizeibeamte streiken? Festschrift zum 13. Delegiertenkongreß der GdP, Bonn 1970, S. 121. 
suchte dieses zunächst, in der Phase des restaurativen Wiederaufbaus in der Bundesrepublik, Zuflucht bei irrationalen Überhöhungen seiner Stellung, um seinen Status durch solche Abgrenzung gegenüber den anderen Arbeitnehmern zu wahren, so daß auch die Beamtengewerkschaften im DGB Beamtenstatus und Beamtenstreik tabuisierten.

Die drei Faktoren: Die Übertragung von immer mehr neuen und neuartigen Aufgaben auf den öffentlichen Dienst, der Einsatz neuer Technologien sowie ein durch die Interessen der privaten Kapitalverwertung bedingter zunehmender Kostendruck seitens der Gesellschaft auf den öffentlichen Dienst, haben seitdem die überkommene Gestalt und Struktur des öffentlichen Dienstes immer stärker in Frage gestellt und den Beamtenstatus verunsichert.

Der besondere individuelle Beamtenstatus beruht auf der Lebenszeitanstellung in Verbindung mit einem allmählichen Aufrücken in Gehalt und Hierarchie nach der Anciennität; die weitgehende Ausschaltung der proletarischen Existenzunsicherheit und die objektiv berechtigte Erwartung und Erfahrung eines individuellen Aufstiegs erzeugten ein bürgerliches Bewußtsein, das sich von der alten "Arbeiterklasse « absetzte. Während die Bedeutung der sozialen Sicherheit in Zeiten der Hochkonjunktur und Vollbeschäftigung ${ }^{14}$ zurücktritt, ja sogar infolge des immanenten, relativen Zurückbleibens der Einkommen der Beamten (bei steigenden Lebenshaltungskosten) durch ein Gefühl der Benachteiligung überdeckt wird, haben die vorgenannten Entwicklungsfaktoren, insbesondere die »Industrialisierung des Büros « durch Mechanisierung, Automatisierung und Rationalisierung, die bürokratischen und hierarchischen Arbeitsformen und -bedingungen so tiefgreifend erschüttert und umgewandelt und in wachsendem Umfang zu Umsetzungen, Qualifikationsverlust, Einschränkung der traditionellen Aufstiegschancen in der Hierarchie und auch zu Freisetzungen geführt, daß ein Gefühl ohnmächtiger Abhängigkeit das bürgerliche Sicherheits- und Aufstiegsbewußtsein der Beamten im Kern trifft und verunsichert ${ }^{15}$. Das bedeutet nicht, daß die Beamten sich einfach als »Proletarier* einzuschätzen beginnen würden. Aber von daher beginnen sie - ähnlich wie auch die Angestellten und die Schicht der technischen Intelligenz in der spätkapitalistischen Gesellschaft ${ }^{10}$ - trotz der fortbestehenden Unterschiede in den Arbeits- und Lebensverhältnissen eine politische und gesellschaftliche Gemeinsamkeit mit den Arbeitnehmern hinsichtlich der Interessenlage am Arbeitsplatz und im öffentlichen Dienst gegenüber dem Dienstherren sowie hinsichtlich der Zugehörigkeit zu den unteren Schichten der Gesellschaft zu entdecken und zu entwickeln.

Beamte, Arbeiter und Angestellte des öffentlichen Dienstes treffen sich in ihren gemeinsamen Interessen an Sicherung gegen Abhängigkeit und an Verbesserung des sozialen Status. Daraus resultiert die Forderung nach einem vereinheitlichten bzw. neuen einheitlichen öffentlichen Dienstrecht, wie sie von den Gewerkschaften (OTV, DGB, DAG) erhoben und von Thieme, Schäfer, Wiese und im Neubauer-Plan aus Berlin aufgegriffen worden ist ${ }^{17}$.

\footnotetext{
11 Wie sie für die Bundesrepublik in den Ietzten Jahren gegeben waren, abgesehen von der schnell wieder aus dem akruellen Bewußtsein verdrängten Rezessionskrise I 966/67.

$15 \mathrm{Vgl}$. Bahrdr, Die Beamten, in: Feuersenger (Hrgb.), Gibt es noch ein Proletariat? 1962, S. $34 \mathrm{ff}$.; Hartfiel, Die öffentliche Verwaltung $\mathrm{zwischen} \mathrm{technischem} \mathrm{Fortschritt} \mathrm{und} \mathrm{sozialem}$ Wandel, Hamburger Jahrbuch für Wirtschafts- und Gesellschaftspolitik, 1970, S. I90 ff.; Rationalisierung und Mechanisierung im öffentlichen Dienst, Gutachten des Instituts für Sozialwissenschaftliche Forschung, I 968 ; auch Benz, S. 64, 67.

16 Vgl. dazu Braun-Fuhrmann, Angestelltenmentalität, 1970, bes. S. 288 ff., s 8 ff.

17 Vgl. OTV-Beamten-Nachrichten Nr. I/70 (S. I) und 8!70 (S. 65 ff.); Grundsätze des Deutschen Gewerkschaftsbundes zur Neuordnung des Beamtenrechts, I 970 (noch zögernd beschränkr
} 
Indem die Forderung nach einem Streikrecht für die Beamten in diesen umfassenderen Rahmen einer angestrebten Gemeinsamkeit der gesellschaftlichen Interessenverfolgung für alle unselbständig und abhängig Beschäftigten einzufügen ist, kann das Beamtenstreikrecht nicht zu einem zusätzlichen parasitären Standesprivileg und Herrschaftsinstrument denaturiert werden - wie es bei der Aussperrung von Studenten durch den "Streik « von Ordinarien geschieht ${ }^{18}$, sondern ist Bestandteil des gemeinsamen Emanzipationskampfes der Unterschichten ${ }^{19}$.

Demgegenüber ist es nicht überraschend, daß die Anerkennung des Beamtenstreikrechts noch keineswegs zur herrschenden Meinung in Rechtswissenschaft und Offentlichkeit geworden ist, vielmehr gerade in der 1970 neu entfachten Diskussion die Zahl der ablehnenden Stimmen überwog. Begründung für die Ablehnung waren meist der Hinweis auf die »h. M.« und deren Argumente aus Artikel 33 Abs. 4 und 5 GG (hergebrachte Grundsätze und Treueverhältnis) ${ }^{20}$. Damit wurde auch die vom Bundesinnenminister offiziell für die Bundesregierung erklärte Ablehnung de lege lata begründet ${ }^{21}$. In Übereinstimmung damit lehnte die öffentlich-rechtliche Abteilung des 48. Deutschen Juristentages in Mainz 1970, deren Gutachter und Referenten ebenfalls - zumindest de lege lata - ein Streikrecht der Beamten verneinten ${ }^{22}$, mit 159 Stimmen gegen 45 bei 34 Enthaltungen ein Beamtenstreikrecht auch für die Zukunft ab. Ebenso verharrt der Deutsche Beamtenbund in der Fassade auf seiner moralischen Ablehnung des Beamtenstreiks, wozu allerdings in bemerkenswerter Diskrepanz sein wiederholtes Drohen mit Protestaktionen mit Auswirkungen auf die dienstliche Ordnung * steht ${ }^{23}$. Zugleich sprachen die der herrschenden Meinung beipflichtenden Außerungen deutlich aus, daß das Streikverbot der letzte und eigentliche Unterschied heute zwischen den Beamten und den Angestellten bzw. Arbeitern des öffentlichen Dienstes ist, d. h. das Streikverbot noch die einzige Rechtfertigung für eine besondere Rechtsstellung der Beamten ist ${ }^{24}$.

Einige Autoren wiederum, die offenbar die traditionellen Argumente der herrschenden Meinung wenig überzeugend finden, suchen die Ablehnung mit neuen Begründungen zu rechtfertigen, so - vor Erscheinen der Gewerkschaftsgutachten - Schwerdtner, der im Hinblick auf eine aus der Lebenszeitanstellung her-

auf das Beamtenrecht mit der Gliederung in Status- und Folgerecht, womit aber ein einheitliches Dienstrecht vorbereitet werden soll, s. S. 7) - dazu aud W. Reuter im *Vorwärts \& vom 29. 10. 70; Die Neuordnung des Offentlichen Dienstes - Ein Denkmodell der DAG, in: $\rightarrow$ Der Angestellte (Ausgabe Der Offentlide Dienst), Nr. 10, Oktober 1970; W. Thieme, Empfiehlt es sich, das Beamtenrecht unter Berücksiditigung der Wandlungen von Staat und Geselischaft neu zu ordnen?, Gutaditen zum 48. DJT 1970, Bd. I, Teil D, S. 64 ff., $72 ;$ F. Sdhäfer, Referat zum selben Thema auf dem 48. DJT 1970, Verhandlungen, Bd. II, Teil O, Seite 10 ff., 17, $26 \mathrm{ff}$.; W. Wiese, Zur Neuordnung des Offentlidien Dienstes, DVBl. 1970, S. $644 \mathrm{ff}$., 650; Vorsdıläge für ein einheitlides Dienstrecht (Schriftenreihe $>$ Verwaltung in der Reform*, hrgb. vom Senator für Inneres, Berlin, Heft 1), 1970.

$18 \mathrm{Vgl}$. Schacht, Einführung durch Aussperrung ? KJ 1968, S. $168 \mathrm{ff}$.

10 Vgl. Däubler, S. 207 ff.; anders aber Ramm, S. 133, 181 (nur »besondere Gruppeninteressen «).

$20 \mathrm{Vgl}$. Maunz a. a. O.; Ule, Entwicklungstendenzen im öffentlidhen Dienst, DVBl. 1970, S. 637 ff., 639; Rehbinder, Besprechung von Däubler, DVBl. 1970, S. 709 f.

21 Antwort auf eine Kleine Anfrage der CDU/CSU-Fraktion, Bundestag-Drudksade Nr. VI/ 1246.

22 W. Thieme, S. 66, 69 (»praktisch nur eine geringe Bedeutung «); F. Schäfer, S. 27 (wegen fehlenden Risikos auf beiden Seiten); Quaritsch, Empfiehlt es sich, das Beamtenrecht unter Berüdssichtigung der Wandlungen von Staat und Gesellschaft neu zu ordnen? Referat ${ }_{4} 8$. DJT 1970, Bd. II, Teil O, S. 34 ff., 48 (Treuepflicht).

2s Vgl. einerseits Krause in *Vorwärts * vom 29. 10. 70 (»trojanisches Pferd «), andererseits Kaises in $*$ Zeit * vom I8. 12.70.

$24 \mathrm{Vgl}$. W. Thieme, S. 70; Bedrohung einer Verfassungsinstitution, hrgb. vom Deutschen Beamtenbund, 1970, S. 9; Rehbinder S. 710 ; Ule, S. 639; Kölble, Grundprobleme einer Reform des öfentlichen Dienstes, DOV I 970, S. 447 ff., 454. 
rührende Risikolosigkeit für die Beamten einen Verstoß gegen die Kampfparität sieht ${ }^{25}$, und Westpfal ${ }^{26}$.

Besonders bemüht sich v. Münch ${ }^{27}$ um weine von der Begründung der h. M. abweichende Argumentation " für das Streikverbot ${ }^{28}$. Die von ihm anerkannten, aber nicht als hinreichend überzeugend erachteten Argumente für ein Streikverbot als hergebrachten Grundsatz (Art. 33 Abs. 5 GG) und aus dem Treueverhältnis, das "demokratisch» begriffen in einer »funktionalen, zugleich rationalen und modernen Sicht « den Vorrang überwiegender Interessen der Allgemeinheit einbeziehe, bringt er in Verbindung mit dem Deprokratiegebot und dem Sozialstaatsprinzip. Gegen das Demolsratiegebot verstoße ein Beamtenstreik, weil er den Vollzug der Gesetzesaufträge des Parlaments, der auch nicht nur zeitweise unterbrochen werden dürfe, verhindere. Kern der Argumentation (»nervus rerum «) ist das Sozialstaatsprinzip, nach dem der Staat existenziell notwendige Leistungen für die Allgemeinheit bereitstellen müsse - wozu alle staatliche Tätigkeit gerechnet wird - mit Vorrang vor individueller Lebensstandardverbesserung und das den Staat verpflichte, »die Behinderung oder Verhinderung der Bereitstellung dieser Leistungen durch Dritte (d. h. auch durch seine Beamten) zu unterbinden *. Dagegen werde in »unmenschlicher * und *unsozialer « Weise verstoßen, wenn infolge eines Beamtenstreiks Renten nicht ausgezahlt würden, Opfer eines Verkehrsunfalls verbluteten oder ein Brand nicht gelöscht würde, zumal regelmäßig die sozial schwachen Schichten stärker betroffen würden. Diese bewußt als »modern « gewollte, scheinbar neue Argumentation läuft jedoch im Kern auf den traditionellen Ursprung der herrschenden Meinung hinaus: das obrigkeitsstaatliche Denken. Die demokratische Vorherrschaft des Parlaments wird nicht in Frage gestellt, weil ein Beamtenstreik den Inhalt der Gesetzesaufträge nicht tangiert und das Parlament auf deren zeitlichen Vollzug in der Regel keinen Einfluß nimmt (auch gar nicht nehmen kann); soweit der Gesetzesvollzug ausnahmsweise die ununterbrochene Kontinuität einer staatlichen Tätigkeit erfordert, wird prinzipiell durch den von den Streikenden eingerichteten Notdienst vorgesorgt. Die bei v. Münch vorausgesetzte Allmacht des Parlaments geht daran vorbei, daß der Staat und sein oberstes Entscheidungsorgan nicht absolute Instanzen sind, sondern in einem gesamtgesellschaftlichen Beziehungsgeflecht stehen - daher die Krise des Parlamentarismus in der spätkapitalistischen Gesellschaft - und der öffentliche Dienst selbst Bestandteil dieser Gesellschaft ist als ein soziales Untersystem, in dem die Bediensteten nicht als bloße Automaten für den Gesetzesvollzug bereitstehen, sondern mit eigenen Rechten ausgestattet sich in einem wechselseitigen Verhältnis zum Staat befinden.

Die Argumentation mit den unmenschlichen, unsozialen Ergebnissen, die sicher jedermann vermeiden will (sogar streikende Beamte), ist in ihrer eingängigen, aber nur scheinbaren Konkretion unmittelbar einleuchtend - und auf Emotionen gerichtet. In ihrer punktuell-konkretistischen Isolierung abstrahiert sie von der gesellschaftlichen Funktion solcher Sozialleistungen im spätkapitalistischen Staat, die nicht nur der Befriedigung von Bedürfnissen dienen, sondern gerade des-

25 Fürsorgetheorie und Entgelttheorie im Recht der Arbeitsbedingungen, I970, S. 94 ff.; dieses Argument weisen Däubler, S. I67 f., Ramm, S. 53 und v. Münch, Rechtsgutachten zur Frage des Streikrechts der Beamten unter besonderer Berüdrsichtigung der Rechtsstellung der Polizeibeamten, 1970, S. 50, unter Hinweis auf die Gebaltseinbußen der Beamten zurück.

26 Streikrecht der Beamten? ZBR 1970, S. $4 \mathrm{ff}$.

27 Rechtsgutachten a. a. O., sowie Beamtenstreik und Sozialstaatsprinzip, ZBR I970, S. 37 I ff.

28 So JZ I 970, S. $66 \mathrm{r}$. 
wegen auch Herrschaftsinstrumente sind. Würde man in v. Münchs Argumentation den Gegenstand staatlicher Tätigkeit, um dessentwillen nicht gestreikt werden dürfe, austauschen (statt Rentenauszahlung etwa die Armenpolizei des vorigen Jahrhunderts), so bliebe der Gedankengang unverändert erhalten, und es zeigt sich die Übereinstimmung des Staatsverständnisses mit der herrschenden Meinung: Der alte Obrigkeitsstaat ersteht neu in Form des autoritären Sozialstaats, der zur sozialen Befriedung gewisse Bedürfnisse befriedigt und damit patriarchalisch bevormundend die Bürger versorgt, ihnen (der Gesellschaft) aber unerreichbar übergeordnet bleibt. Wenn auch v. Münch in diesem Sinne das "soziale Wohl der in dem Staat lebenden Menschen " berücksichtigt, so bleibt doch »der Staat $\alpha$, dem gegenüber die Beamten ebenso wie die Bürger als Leistungsempfänger nur außenstehende $» D$ ritte sind, als hoheitlicher Machtapparat von der Gesellschaft losgelöst. Indem die Beziehungen zwischen Beamten, Leistungsempfängern und »dem Staat« nicht als sozialer Wirkungszusammenhang erkannt und in die rechtliche Betrachtung einbezogen werden, bleiben die konkreten gesellschaftlichen Herrschaftsfunktionen der spätkapitalistischen Staatsgewalt außen vor. Demgegenüber muß der Beamtenstreik als Teil der Emanzipationsbewegung der Arbeitnehmer und der sozialen Unterschichten überhaupt begriffen werden, in deren Rahmen die Solidarität mit den sozial Schwachen durch den Notdienst der Streikenden organisiert wird, die auch für die bei v. Münch herausgestellten Einzelfälle Vorsorge trifft - nach der historischen Erfahrung im Ernstfall zuverlässiger als der vorwiegend von anderen Interessen bestimmte «Staat« der spätkapitalistischen Gesellschaft.

Die neuaufgelebte Diskussion hat den Prozeß der Angleichung des Beamtenverhältnisses an das allgemeine Arbeitsverhältnis verstärkt ins Bewußtsein gehoben, insbesondere bei der Begründung des Streikrechts auch für Beamte und der Widerlegung der Argumente der ablehnenden herrschenden Meinung, aber auch wenn $v$. Münch wenigstens die allzu offensichtlich mit dem monarchischen Obrigkeitsstaat verknüpften Begründungen für ein besonderes Wesen des Beamtenverhältnisses - wie Fehlen der Polarität zwischen Arbeitnehmer und Arbeitgeber, Verzicht der Beamten usw. - ablehnt ${ }^{29}$.

Die juristische Aufarbeitung dieses Angleichungsprozesses bestimmt maßgeblich die Auslegung von Art. 33 Abs. 4 und 5 GG. Die dort verankerte Treuepflicht des Beamten entspricht der von der Rechtsprechung formulierten Treuepflicht des Arbeitnehmers und steht einem Streikrecht nicht entgegen, weil sie anstelle der früheren personalen, heute nur irrational möglichen Bindung nur noch in msäkularisierter * Gestalt wie die Treuepflicht des Arbeitnehmers als Verpflichtung zur Förderung der Belange des Dienstherrn verstanden werden kann, ohne Interessenkonflikte aufheben zu wollen ${ }^{30}$. Auch über die »hergebrachten Grundsätze « des Abs. 5 kann ein Streikverbot nicht aus dem Grundgesetz abgeleitet werden, weil zum einen das Streikrecht nicht das Wesen des Berufsbeamtentums berührt, vor allem aber wegen der inzwischen eingetretenen oder wenigstens drohenden Benachteiligung der Beamten ein Festhalten am tradierten Streikverbot den Schutzzweck des Art. 33 Abs. s GG verkehren und die ganze Institution in Frage stellen würde ${ }^{31}$.

Denn Benz hat klar herausgearbeitet, daß das Beamtenverhältnis wegen seiner

20 Gutachten S. 44 ff.; vgl. Däubler, S. I 27 ff.; Benz, S. 16 ff.

${ }^{30}$ Vgl. Däubler, S. I Is ff.; Benz, S. 26 ff.; Ramm, S. 46.

31 Vgl. Däubler, S. 19, I03 ff., III ff., 227 ff.; Benz, S. I 50 ff.; Ramm, S. 19, 35 f., 39, 48, 104 f.; Cech, Der Streik ion öffentlichen Dienst nach französischem und deutschem Redht, Diss. Göttingen 1970, S. 78,83 f.; auch Schwerdener, S. 95 f. 
kollektivrechtlichen Schwäche heute nicht mehr unvergleichliche, exklusive Privilegien und nicht mehr den notwendigen Schutz der Beamteninteressen bietet: $\mathrm{Da}$ das Arbeitsrecht, führend in der Rechtsentwicklung, große Fortschritte gerade auch im Hinblick auf die früheren Privilegien der Beamten gemacht und das Beamtenrecht stagniert hat, sind in dem Einebnungsprozeß aus dem früher generellen Unterschied nur noch graduelle Abweichungen geworden, und sind die Beamten individualrechtlich zurückgefallen trotz Aufrechterhaltung eines erhöhten Pflichtenstatus - infolge ihrer kollektiven Schwäche ${ }^{92}$. Wenn auch diese Beurteilung $\mathrm{zu}$ formalistisch uibersieht, daß die Lebenszeitanstellung $\mathrm{zwar}$ nicht mehr als exklusives Privileg nur der Beamten besteht und vermittels Unkündbarkeit auch bei Arbeitnehmern möglich ist, aber für die Beamten weiterhin die Regel darstellt und für die Arbeitnehmer seltene Ausnahme ist, so trifft sie doch die reale Tendenz zur Herstellung einer Gemeinsamkeit von Beamten- und Arbeitsverhältnis durch beidseitige Anhebung auf eine für alle bessere Ebene gerade auch im Hinblick auf die gewerkschaftliche Forderung nach einem effektiven individuellen Recht auf Arbeit und am Arbeitsplatz für alle Arbeitnehmer, was das Pendant zur Lebenszeitanstellung darstellt (und nur als systemüberwindende Reform möglich ist).

Daran zeigt sich, daß das Streikreicht »nur einen Teil des Gesamtproblems der Auffassung vom Beamtenverhältnis « bildet, das im demokratischen Sozialstaat des Grundgesetzes nur ein freiheitliches Rechtsverhältnis als gegenseitiges Vertragsverhältnis sein und keiner grundsätzlichen rechtlichen Sonderbewertung unterworfen werden kann. Auch Beamte sind Arbeitnehmer, und das Beamtenrecht ist Teil des Arbeitsrechts, wie Ramm gerade aus der Interpretation der allumfassenden Koalitionsfreiheit des Art. 9 Abs. 3 GG begründet ${ }^{33}$. Dementsprechend ist die beide Bereiche integrierende Reform zu einem einheitlichen öffentlichen Dienstrecht im Sinne der oben genannten Vorschläge ${ }^{34}$ mit dem Grundgesetz vereinbar ${ }^{35}$ - wobei allerdings die im Vergleich mit der Privatwirtschaft bestehende Besonderheit des öffentlichen Dienstes berücksichtigt werden muß und kann.

Die inhaltliche Bestimmung dieser Besonderheit hängt ab von dem zugrunde liegenden Staatsverständnis, das überhaupt für die gesamte Diskussion über den Beamtenstreik tonangebend ist. Das Staatsverständnis der Verteidiger des Streikverbots hat zuletzt Quaritsch ${ }^{36}$ vorgetragen: „Der Moderne Staat « der Neuzeit, der nach Quaritsch als eine typisch wunkapitalistische Erscheinung in Ost und West ungeachtet der grundverschiedenen Gesellschaftssysteme bestehe, beruhe auf der Trennung von Staat und Gesellschaft, von politischer und privater Sphäre. Das fundamentale und konstitutive Kriterium für die Eigenart »des Modernen Staats«, der als solcher in jeder staatlichen Tätigkeit präsent sei, sei die Befugnis zu autonomen, einseitigen Entscheidungen, ohne vom Plazet gesellschaftlicher Größen abhängig zu sein, was eine Beeinflussung von jener Seite nicht ausschließe. Dieser »Moderne Staat« stehe und falle mit dem Status seiner Bediensteten, der gemäß der $n$ Logik des Modernen Staates « ebenfalls einseitig vom Staat her zu bestimmen sei ${ }^{37}$.

32 Vgl. Benz, S. I f., 16 ff., so ff., I09, I48 f.; Ramm, S. 17, 23, 26, 37, 70; Däubler, S. 185 , 2II f., 217

ss Vgl. Ramm S. 23 f., 37, 54, I15; Benz, S. 148.

34 s. Anm. 17.

$95 \mathrm{Vgl}$. auch Wiese S. 650 f.; a. A. Ule a. a. O.

36 S. 35 ff., 45, 52; ebenso Ule S. 639; vgl. auch das oben zu v. Münch (nach Anm. 28) Gesagte.

s7 Im Grunde das gleiche Staatsverständnis kommt auch bei Ramm, S. 40, 44, I4I ff., und Benz,

S. 128 f., I 43 ff., zum Vorschein, wenn sie einerseits in differenzierender Betrachtung das Streik- 
Diese Vorstellung von dem einen "Modernen Staat* ist nun eine typische kapitalistische Ideologie, gerade weil sie den gegenwärtigen Staat der Bundesrepublik als typisch »unkapitalistische Erscheinung von seinen konkreten gesellschaftlichen Bedingungen abzuheben unternimmt. Denn erstens ist der Staat in der Neuzeit zugleich und in unmittelbarer innerer Verbindung mit der kapitalistischen Gesellschaft und deren Funktionsbedingungen herausgebildet worden. Zweitens gibt es "den* neuzeitlichen Staat als einheitliche Kategorie jedenfalls insoweit nicht mehr, als inzwischen auch andere, nicht-kapitalistische Gesellschaften mit fundamental anderen Strukturen des gesellschaftlichen Reproduktionsprozesses entstanden sind, in denen die Funktionen des Staats infolge der grundlegend andersartigen gesellschaftlichen Bedingungen wesentlich anders definiert sind und organisiert werden. Der Staat der Bundesrepublik unterliegt als wesentlicher Bestandteil des gesellschaftlichen, d.h. spätkapitalistischen Reproduktionsprozesses dessen Strukturbedingungen und damit auch der gesellschaftlichen Vorherrschaft der durch ihre Verfügungsgewalt über die Produktionsmittel definierten Klasse. Wenn mit der Behauptung einer autonomen Einseitigkeit der staatlichen Entscheidung deren Bedingtheit und Abhängigkeit von den konkreten gesellschaftlichen Kräfteverhältnissen geleugnet wird, so dient eine solche Verschleierung der realen Vorherrschaft der kapitalistischen Interessen (nicht nur bei der "Beeinflussung « auf die, sondern direkt innerhalb der staatlichen Willensbildung) gerade der Legitimation dieser kapitalistischen Herrschaft.

Ist daher der Staat insgesamt als ein sozialer Wirkungszusammenhang zu erkennen, so ist auch das Beamtenverhältnis eine besondere wechselseitige Beziehung, in der nach den grundgesetzlichen Prinzipien des demokratischen Sozialstaates »der Staat« weder als Hoheitssubjekt noch als Arbeitgeber einseitig den Status begründen und bestimmen darf. Lediglich die Volkssouveränität gebietet einschränkend, daß die Bestimmung von Aufgabe und Gegenstand der staatlichen Tätigkeit allein dem Parlament vorbehalten bleibt und nicht von den Bediensteten, die - jedenfalls unter den Bedingungen der kapitalistischen Gesellschaft - bei der Behandlung dieser Fragen nicht so sehr die gemeinsamen allgemeinen Interessen von Arbeitnehmern und Beamten, sondern vorwiegend ihre gruppenspezifischen Interessen vertreten, mitentschieden werden kann. Allein darin, daß die Bediensteten nicht auch über die Aufgabe des öffentlichen Dienstes, $d . h$. über den Inhalt ihrer Arbeit insgesamt - wie zumindest theoretisch denkbar die Arbeitnehmer in der Privatwirtschaft über den Unternehmensgegenstand und -zwedk - mitbestimmen dürfen, liegt die anzuerkennende Besonderheit des öffentlichen Dienstes, die bei der Gestaltung eines neuen einheitlichen öffentlichen Dienstrechts zu beachten ist ${ }^{38}$. Wegen dieser Besonderheit kann das einheitliche Dienstrecht unschwer nach der Subjekt- und Interessentheorie dem öffentlichen Recht (Art. 33 Abs. 4 GG) zugeordnet werden, nachdem die nach der Subjektionstheorie vorgenommene Abgrenzung zwischen öffentlichem und Privatrecht infolge der Relativierung der ihr zugrunde liegenden Trennung von Staat und Gesellschaft allgemein fragwürdig geworden ist und die Abgrenzung heute nur organisierende Bedeutung hat, ohne daß bereits mit der

recht nur für bestimmte Funktionen des öffentlichen Dienstes zulassen bzw. ausschließen und andererseits (nur) eine Reduzierung des Beamtenverhältnisses (letztlich unter Beibehaltung der alten Gestalt mit Streikverbot) auf den sUrsprungs- und Kernbereich staatlicher Betätigung (d. i. s Sicherheitsschaffung nach außen und innen *) befürworten.

38 Vgl. auch Däubler, S. I60 f. 
Zuordnung zu einem der beiden Rechtsgebiete vorweg materiell-inhaltliche Wertungen getroffen würden ${ }^{30}$.

Deswegen steht in einem einheitlichen Dienstrecht dem Abschluß von Tarifverträgen ebensowenig entgegen wie schon heute einem Beamtentarifvertrag, was Däubler ${ }^{40}$ überzeugend de lege lata aus Art. 9 Abs. 3 GG abgeleitet hat, der als Garantie der spezifischen Koalitionsbetätigung gerade auch den Abschluß von

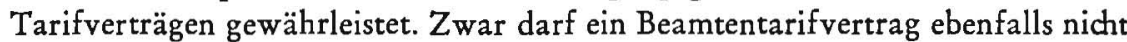
in die gesetzlich festgelegte Aufgabenstellung des öffentlichen Dienstes eingreifen, aber die im Grundgesetz verankerte sozialstaatliche Demokratie gebieter keineswegs eine umfassende gesetzliche Regelung des gesamten Dienstrechts, wie sie vom Bürgertum in seiner Auseinandersetzung mit dem monarchisch-feudalistischen Staatsapparat durchgesetzt worden war, als das Gesetz noch der einzige Weg zur Beschränkung einer nicht demokratisch legitimierten Staatsgewalt war. Soweit daher beamtenrechtliche Gesetze als abschließende Regelungen nicht bloß Mindestbedingungen enthalten und kollektivrechtliche Regelungen ausschließen wollen, verstoßen sie gegen Art. 9 Abs. 3 GG. Daraus ergibt sich wiederum der regelungsfreie Raum, der durch Tarifvertrag mit Hilfe des Streiks ausgefüllt werden kann, welcher dann kein sog. "politischer" Streik ist, weil der Gesetzgeber nicht mehr Adressat ist. Derart kann die gewerkschaftliche Forderung nach Abschluß eines Beamtentarifvertrags zum Hebel werden für die Durchsetzung des Streikrechts und die Fortentwicklung des Beamtenrechts.

Nach Däubler, Benz und Ramm steht nach dem Grundgesetz jedermann das Streikrecht zu, damit auch den Beamten. Diese grundgesetzliche Streikgarantie wird allerdings unterschiedlich begründet, nämlich entweder aus Art. 9 Abs. $3^{41}$ oder, zumindest primär, aus Art. 2 Abs. I (in Verbindung mit Art. 19 Abs. 3$)^{42}$.

Die unterschiedliche Begründung ist zugleich bedeutsam für die Begrenzung des Streikrechts, die für Däubler nach Art. 9 Abs. 2 GG lediglich - abgesehen von den hier nicht relevanten *allgemeinen « Strafgesetzen - in der verfassungsmäßigen Ordnung, verstanden als Inbegriff der strukturierenden Grundprinzipien der Verfassung, besteht, während $\mathrm{Ramm}^{43}$ die Schrankentrias des Art. 2 Abs. I GG anwendet, wobei er die verfassungsmäßige Ordnung ebenso eng wie Däubler versteht; aber als "Rechte anderer " die dem Individuum unter Ausschluß Dritter gewährten Herrschafts- und Schutzbereiche begreift und dem "Sittengesetz « die freiheitliche soziale Grundordnung des Grundgesetzes unterordnet. Diese Grenzen wirken sich auch in dem Verbot bestimmter Streiks aus, die aber - entgegen der herrschenden Meinung zum Beamtenstreikverbot - nicht nach dem persönlichen Rechtsstatus des einzelnen abgegrenzt werden, sondern durch eine Differenzierung nach den (staatlichen) Funktionen, die die Bediensteten jeweils wahrnehmen ${ }^{44}$. Soweit danach der Streik für in bestimmten Funktionen Beschäftigte ausscheidet, soll eine "sozialstaatliche Gestaltung ", die institutionell sicherstellen soll, daß auch diese Bediensteten trotz Streikverbots gleichermaßen ihre Arbeits- und Lohnbedingungen verbessern können, in ihrer gewerkschaftlichen Zusammenfassung mit anderen Arbeitnehmern bestehen, so daß de-

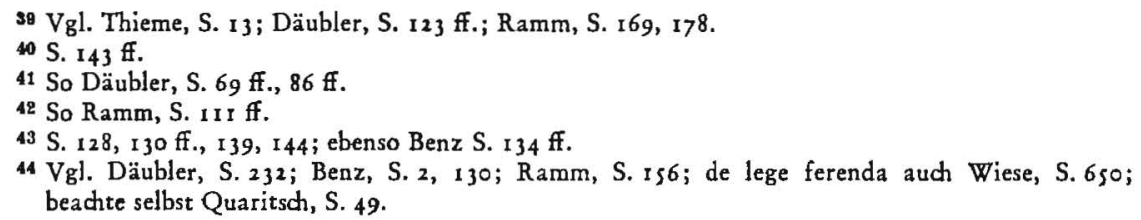


ren Tarifvertrag automatisch auf die nicht-streikberechtigten Bediensteten erstreckt werden können ${ }^{45}$.

Eine Anwendung dieser Grundsätze auf den gesamten staatlichen Tätigkeitsbereich läßt eine starke Differenzierung zwischen den einzelnen Funktionen erkennen, für die entweder eine weitgehende oder eingeschränkte Streikfreiheit oder auch ein absolutes Streikverbot gelten soll, wobei sich die einzeinen Autoren keineswegs immer einig sind ${ }^{46}$. Das Gesamtbild ergibt eine ganz beachtliche Zahl von Einschränkungen für den "Beamtenstreik «, der jedoch nach Meinung der Gutachter nicht zu einer Ausnahme werden sollit7. Eine nüchtern-realistische Betrachtung muß allerdings den Wert dieser differenzierenden Meinungen mit ihren so diffizilen Abwägungen für den Einzelfall für eine praktische Nutzanwendung durch Beamtengewerkschaften bezweifeln. Solche Zweifel sind um so begründeter, als diese differenzierenden Einschränkungen des Streikrechts nicht nur für Beamte, sondern auch für alle Arbeitnehmer des öffentlichen Dienstes und gar der Privatwirtschaft gelten sollen - deren Streikrecht bislang einer solchen Einschränkung nicht unterworfen worden ist -, sowohl wenn eine öffentliche Aufgabe privatrechtlich-privatwirtschaftlich organisiert wird, als auch generell für den gesamten Bereich der Privatwirtschaft ${ }^{48}$. Die Ưbertragung der funktionalen Differenzierung zwischen Streikfreiheit und Streikverbot auf den (gesamten) arbeitsrechtlichen Bereich trifft sich mit der neuerlich im Vordringen begriffenen Meinung, die entgegen der bislang herrschenden Meinung ein generelles Streikverbot für lebensnotwendige Versorgungsbetriebe behauptet ${ }^{49}$. Darin liegt eine gefährliche Einschränkung des Streikrechts, weil sie inhaltlich nicht fest abzugrenzen ist und zu befürchten ist, daß letztlich alle Betriebe und Funktionen der lebensnotwendigen Versorgung der Bevölkerung zugerechnet werden, wie es die Parallele in $\$ 4$ Abs. 2 Arbeitssicherstellungsgesetz bereits andeutet.

Deswegen hat der OTV-Vorsitzende Kluncker auf einer Pressekonferenz am 20. 4. 70 das Gutachten von Däubler auch "mit einem weinenden Auge « betrachtet, weil nach dessen Ergebnissen »das Streikrecht eine mehr oder weniger akademische Sache bliebe* und durch die Einbeziehung der Arbeiter und Angestellten (z. B. der gewerkschaftlich gut organisierten Müllarbeiter) in das funktional begründete Streikverbot in der Praxis der Streik unwirksam gemacht zu werden drohe. Uber diese Zusammenhänge hat die Diskussion um den Beamtenstreik gerade mit den Gewerkschaftsgutachten deutlich die Gemeinsamkeit des Teilproblems mit dem Streikrecht aller Arbeitnehmer herausgestellt. Die Frage nach Streikrecht oder Streikverbot kann nicht mehr isoliert für die Beamten als eine begrenzte Gruppe mit besonderer Rechtsstellung gestellt werden, sondern muß nach dem gegenwärtigen Diskussionsstand im Beamtenrecht wie im Arbeitsrecht folgerichtig generell als Frage nach dem Inhalt des Streikrechts für alle »Lohn-

45 Vgl. Ramm, S. I70 ff.; auch Benz, S. I42; จ. Münch, Gutachten, S. 9 I ff.

16 Vgl. Däubler, S. 232 ff.; Ramm, S. I 30 ff.; Benz, S. I 33 ff. Ramm (S. I 48 ff., Is6, Is8, I6I, 170) redet außerdem noch von einem pfliditgemäßen (und bezahlten) Streik im allgemeinen Interesse gegen unhaltbare Mißstände, die der Realisierung des konkreten Verwaltungszwecks, etwa des Rechts auf Bildung oder des Schutzes der Volksgesundheit, entgegenstehen; der praktische Nutzen einer solchen Kategorie, die nicht als Recht zur Einwirkung auf die Gestaltung der Arbeitsbedingungen gedacht ist und in die Nähe des Widerstandsrechts rüdkt, ist nicht zu erkennen.

47 Vgl, Ramm, S. Iss; Däubler, S. 240 f.; zweifelnd aber Löwisch, DOV 1970, S. 687.

48 Vgl. Ramm, S. I19, I25, 129, I 47, I 56; Benz, S. I 32 ff.; Däubler, S. 233 ; Cech, S. 95 , I Is.

40 Vgl. Brox-Rüthers, Arbeitskampfrectit, 1965, S. 69, I 50; zur h. M. siehe Huedk-Nipperdey, Lehrbuch des Arbeitsrechts, II/2, 7. Aufl. 1970, S. 984, 1030 Anm. 83: Streikverbot nur, wenn keine gewerkschaftlichen Notarbeiten. 
abhängigen « (cinschließlich der Beamten) sowie nach den Möglichkeiten einer staatlichen Einschränkbarkeit gestellt werden. ${ }^{50}$

Auch für die Gewerkschaften ist es selbstverständlich, daß ein Streik Grenzen hat. Dementsprechend ist in den verbindlichen Arbeitskampfrichtlinien des DGB bei Streiks in lebenswichtigen Betrieben eine gewerkschaftliche Verpflichtung auf Notstandsarbeiten zur Sicherung der Versorgung der Bevölkerung ausgesprochen ${ }^{51}$. Die entscheidende, vom Grundgesetz her zu beantwortende Frage ist aber: wer bestimmt solche Grenzen und wer wacht über ihre Einhaltung, der Staat gegenüber den Gewerkschaften oder die Gewerkschaften selbst, die durch Notstandsarbeiten eigenverantwortlich die Grenzen einhalten? Der Notdienst bei Streiks ist auch in den Gutachten zum Beamtenstreik für die Feststellung der Streikfreiheit einbezogen worden, deren Ausdehnung in den einzelnen Staatsfunktionen jeweils in Abhängigkeit von der Existenz und Möglichkeit eines Notdienstes bestimmt wurde ${ }^{52}$. Dabei ist allerdings nicht ausdrüdklich gesagt worden, ob insoweit noch ein Eingriffsrecht des Staates besteht, oder ob mit dem Notdienst die Gewerkschaft in eigener Verantwortung und ohne staatliches Eingriffsrecht die Grenzen des Streiks bestimmen kann. Die Formulierung bei Ramm, daß der Schutz der notwendigen staatlichen und gesellschaftlichen Funktionen von den Gewerkschaften im Rahmen ihrer satzungsmäßig vorgesehenen Notdienstverpflichtung übernommen werden könne, könnte auf eine staatsfreie Verantwortung der Gesellschaft hindeuten. Jedoch lassen die Ableitung von Grenzen des Streikrechts, als Grenzen der verfassungsrechtlichen Gewährleistung des Streikrechts, aus den für Staatsbefugnisse konstitutiven Schranken des Art. 2 Abs. I bzw. Art. 9 Abs. 2 GG sowie die Anerkennung einer Befugnis des Gesetzgebers, das Streikrecht zu regeln und einzuschränken, erkennen, daß die staatlichen Instanzen das letzte Eingriffsrecht haben und letztlich die Grenzen des Streikrechts festlegen sollen ${ }^{53}$. Demnach würden die Grenzen des Streiks vom Gesetzgeber als Grenzen der Verfassungsgarantie des Streikrechts bestimmt.

Demgegenüber ist jede einschränkende Fremdbestimmung über Inhalt und Ausdehnung des Streikrechts der Arbeitnehmer und Gewerkschaften abzulehnen, auch seitens »des Staats «, weil sie im Grundgesetz nicht vorgesehen ist und allein schon das prinzipielle Zugeständnis einer staatlichen Kompetenz zur *Aufsicht « bzw. Grenzziehung durch den Gesetzgeber mangels einer eindeutigen inhaltlichen Beschränkung, die durch derart unbestimmte Begriffe wie wverfassungsmäßige Ordnung « nicht geleistet wird, bis hin zu einer praktischen Unwirksamkeit des Streikrechts ausgenutzt werden könnte (je nach den konkreten gesellschaftlichen Machtverhältnissen). Vielmehr muß es allein der ausschließlichen

\footnotetext{
50 Während nach allgemeiner Meinung für die Soldaten ein prinzipielles Streikverbot gilt (vgl. Ramm, S. I5s; Benz, S. 135; Däubler, S. 3; Maunz-Dürig-Herzog, Rn. I33 mir der merkwürdigen Einschränkung auf rregelmäßig*), scheint der Streikbazillus auch diese Gruppe angesteckt zu haben, s. "Spiegel Nr. 48 vom 23. I I. 70. In einer Bundeswehr, die nicht mehr monolithisch von oben nach unten, sondern auf der individuellen Verantwortung für die Befehlausführung ( $\$$ II Soldatengesetz) aufgebaut ist, kann auch der Streik kein Fremdkörper mehr sein. Da die soziale Abhängigkeit der Soldaten der der Beamten vergleichbar ist und insoweit kein grundsätzlicher Unterschied zwischen Berufssoldaten und Wehrpflichtigen besteht (trotz des unterschiedlichen Unterhalts, vgl. W. Wiese, Der Streit um die Alimentationstheorie, VerwArch. 1966, S. 240 ff., 246, 252), muß der Streik aud für Soldaten erlaubt sein, (entgegen meiner früheren Ansicht in AöR 1966, S. 187 Anm. 241). Erst recht ist ein Streik der Ersatzdienstpflichtigen zulässig, entgegen der offziellen Ansicht lt. „Frankfurter Rundschau* vom S. 12.70.

61 RdA i 950, S. 7 r f.

52 Vgl. Däubler, S. 233, 236, 239; Ramm, S. 157, I8I, I85; Benz, S. I 39.

33 Vgl. Ramm, S. I19, I35; Däubler, S. I39.
} 
Eigenverantwortung der Arbeitnehmer und ihrer Gewerkschaften überlassen bleiben - ohne eine staatliche Uberwachung und Kompetenz-Kompetenz -, die Grenzen des Streiks festzulegen und einzuhalten durch die Notstandsarbeiten der Streikenden, die als Bestandteil der Kampfmaßnahmen nur unter gewerkschaftlicher Kontrolle der Streikleitung, ohne Mitspracherecht der Geschäftsleitung, ausgeführt werden ${ }^{54}$. Die gewerkschaftlichen Notstandsarbeiten, die gerade die staatliche Intervention in die Arbeitskampfsphäre ausschließen sollen, organisieren die gesellschaftliche Solidarität unter und zwischen den Arbeitnehmern bzw. sozialen Unterschichten, also der ganz überwiegenden Mehrheit der Bevölkerung. Solche Solidarität ist wesensnotwendige Voraussetzung jeder gewerkschaftlichen Betätigung, die ohne Solidarität mit gesamtgesellschaftlichem Bezug nicht denkbar und möglich ist, und funktioniert auch wirklich nach der historischen Erfahrung, wie etwa das Beispiel des Berliner Arbeiter- und Soldatenrats 1918 zeigt, der ausdrücklich die lebenswichtigen Betriebe vom Generalstreik ausschloßss. Die Ablehnung einer staatlichen Kompetenz für die Beschränkung des Streikrechts begründet nicht eine rechtsfreie Sphäre, sondern schützt nur eine staatsfreie Sphäre, die vom Verfassungsrecht eingeräumt worden ist, deren Grenzen sich aus ihrem Gegenstand selbst ergeben und als solche vom Recht anzuerkennen sind.

Mit dem Grundgesetz hat der Verfassungsgeber als pouvoir constituant in dem gleichen Akt, mit dem er erst die Staatsorgane und deren Befugnisse schuf, auch diese Handlungsmöglichkeit der Arbeitnehmer und Gewerkschaften anerkannt und durch deren Ausschluß aus der Kompetenz der Staatsorgane die konkrete Staatlichkeit des Grundgesetzes bestimmt. Denn Art. 9 Abs. 3 GG enthält die Garantie des Streikrechts, ohne daß vom Grundgesetzgeber her besondere, einschränkende Grenzen zugelassen wurden. Insbesondere kann nach den anerkannten Methoden systematischer Auslegung nicht ersatzweise Art. 9 Abs. 2 GG herangezogen werden, der nur für das andersartige Grundrecht der Vereinigungsfreiheit gilt und gerade infolge seiner systematischen Stellung den Abs. 3 nicht betrifft ${ }^{56}$. Eine solche Freiheit von staatlichen Begrenzungen hat auch ihren konkreten sozialen Sinn: Ein derart von staatlichen Schranken freigestelltes Streikrecht kann nicht zu schrankenloser, willkürlicher Allmacht der Arbeitnehmer und Gewerkschaften führen, sondern wird von vornherein begrenzt durch die gesellschaftliche Realität der ihnen gegenüberstehenden, überlegenen Machtpositionen der herrschenden Klasse. Um die von dem politischen Einfluß der sozialen Vorherrschaft der Kapitalinteressen drohenden staatlichen Einschränkungen des Streikrechts abzuwehren, hat das Grundgesetz zur Sicherung der Gegenmachtposition der Arbeitnehmer in dem von ihm gewollten demokratischen Sozialstaat das Streikrecht der staatlichen Kompetenz entzogen.

Die Sozialstaatsklausel des Grundgesetzes wird bislang als "Staatszielbestimmung " zwar formal hoch eingeordnet, aber nur mit einem reichlich unbestimmten Inhalt. In der Praxis ist sie lediglich in einer allgemeinen »wohlfahrtsstaatlichen * Richtung operationalisiert worden, zur Unterstützung und zum Ausbau

34 So Lauschke, Die Notarbeiten im Arbeitskampf als kollektivrechtliche Maßnahme, DB 1970 , S. I17s ff.; entgegen LAG Frankfurt AuR 1970, S. 349 ff. m. Anm. Herschel.

35 S. dazu Ramm, S. 75.

so Die bei Däubler, S. 86, offengelegte Motivation für das Bemühen um die Konstruktion besonderer Schranken für das Streikrecht vermag nicht zu überzeugen: die Gefahr einer wtotalen Verabsolutierung \& geht von falschen Voraussetzungen aus, s. dazu weiter im Text, während der Versuch, die sonst drohende Konstruktion simmanenter \& Grenzen etc. durch eigene einschränkende Interpretation zu vermeiden, als Taktik der Wahl des $\$$ kleineren Ubels* abzulehnen ist, weil sie vorschnell Rechte preisgibt. 
von Leistungsansprüchen der einzelnen an den Staat. Da hierbei die Verbindung zur demokratischen Forderung nach einer gleichberechtigten Beteiligung möglichst aller an dem gesellschaftlichen und politischen Entscheidungsprozeß nicht hergestellt wird, ist darin die bereits oben erwähnte Tendenz zum autoritären Sozialstaat angelegt. Anstelle einer solchen Verengung des Sozialstaatsprinzips gilt es, eine bislang vernachlässigte Komponente der Sozialstaatlichkeit zu entfalten, die sich unter Bezugnahme auf Art. 20 Abs. I GG aus einer Zusammenschau vom »demokratischen und sozialen (Bundes-)Staat « ergibt ${ }^{57}$. Danach steht das Sozialstaatsprinzip in verfassungsgeschichtlichem Zusammenhang mit der sozialen Bewegung seit dem I 9. Jahrhundert, deren Ideengut und Praxis in ihrer sozialreformerischen wie in ihrer sozialrevolutionären Tendenz über die Sozialstaatsklausel in das Grundgesetz aufgenommen worden ist. Die soziale Bewegung als Protest der Massen zeichnet sich einerseits durch ihr Streben nach einer "sozialen Demokratie « aus, die letztlich das Selbstyerwaltungsprinzip auf den gesamten gesellschaftlichen Bereich ausdehnen und in Rüdkwirkung auf den staatlichen Bereich die staatliche Demokratie vervollständigen sollte, andererseits durch die - damit zusammenhängenden - Formen ihres letztlich auch staatsbezogenen, politischen Handelns durch kollektive Interessenvertretung außerhalb der staatlichen Institutionen, von deren Funktionieren sie zunächst ausgeschlossen gewesen war.

Diese Charakteristika der sozialen Bewegung sind auch noch für den gegenwärtigen Staat der spätkapitalistischen Gesellschaft bedeutsam, dessen Wirkungszusammenhang als politische Einheit ebenfalls nicht auf einer substantiellen Homogenität des Volkes beruht, sondern infolge der Klassenspaltung der Gesellschaft von unterschiedlichen sozialen Interessen und Interessengegensätzen bestimmt wird. Da der spätkapitalistische Staat ein wesentlicher Bestandteil des gesellschaftlichen Reproduktionsprozesses ist und die politische Gewalt die Verteilung der gesellschaftlichen Güter vermittelt, sind die sozialen Interessengegensätze unmittelbar in das politische Institutionensystem einbezogen. Als Vermittlung der sozialen Interessen jedoch reicht das System parlamentarischer Repräsentation nicht aus, weil es auf einer nur formalen staatsbürgerlichen Gleichheit beruht, die nur dadurch zustande kommt, daß von den individuellen sozialen Interessen und der sozialen Ungleichheit abstrahiert wird, die also auch nicht unmittelbar in den parlamentarischen Repräsentationsprozeß eingehen können. Andererseits definieren die vorgegebenen sozialen Machtpositionen, über welche die durch ihre Verfügungsgewalt über die Produktionsmittel vorherrschende kapitalistische Klasse bestimmt, den Handlungsrahmen der in dem parlamentarischen Repräsentationsprozeß konstituierten Staatsorgane. Um eine freiheitliche Demokratie und einen demokratischen Sozialstaat zu bewahren, müssen die gesellschaftichen Einwirkungen auf die Staatsgewalt als freiheitsverbürgendes Korrektiv wirken. Dies ist nicht gewährleistet, solange nur die sozialen Machtpositionen der Unternehmerseite allein schon aufgrund ihrer faktischen Existenz als bestimmende $\mathrm{Da}$ ten der politischen, staatlichen Entscheidungsbildung fungieren. Nur dann, wenn nicht nur die Unternehmer, sondern auch die Arbeitnehmer die spezifischen sozialen Aktionsformen, die sich aus den sozialen Bedingungen ihrer Klasse erge-

$57 \mathrm{Vgl}$, zum Folgenden Abendroth, Das Grundgesetz, I966, S. 64 ff.; Ridder, Zur verfassungsrechtlichen Stellung der Gewerkschaften im Sozialstaat nach dem Grundgesetz, I960, S. S, I I ff., 30 f.; Lenz, Die unbehagliche Nähe der Koalitionsgarantie zum Sozialstaat, in: Gesellschaft, Recht und Politik (Festschrift Abendroth), I 968, S. 203 ff.; Ramm, Der Arbeitskampf und die Gesellschaftsordnung des Grundgesetzes, I965, S. I44 ff.; U. Preuß, Zum staatsrechtlichen Begriff des Offentlichen, 1969, S. 131 ff., $163 \mathrm{ff}$. 
ben, staatsbezogen auf den politischen Entscheidungsprozeß einwirken lassen können, besteht tatsächlich ein freiheitsverbürgendes Korrektiv. Das spezifische, aus ihrer Klassenlage resultierende Aktionsmittel der Arbeitnehmer aber ist der Streik, der schon in der Vergangenheit als ständige "Begleiterscheinung « gesellschaftlicher Umbrüche im Dienste der freiheitlichen Emanzipation und der Menschenwürde eingesetzt wurde ${ }^{68}$. Indem das Sozialstaatsprinzip, das seiner Intention nach gegen das Eigentum an Produktionsmitteln und dessen Machtausdehnung gerichtet ist, den Streik der Arbeitnehmer als Korrektiv gegen die sozialen Machtpositionen der Unternehmerseite umfassend anerkennt und von staatlicher Einschränkung freihält, liegt in ihm der Verfassungskompromiß des Grundgesetzes begründet, der die legale Möglichkeit einer grundlegenden gesellschaftlichen Umgestaltung im Rahmen des Grundgesetzes offenhält.

Gegen diese sozialstaatliche »Privilegierung « des Streikrechts wird der Vorwurf des wantidemokratischen « Rüdkfalls in den "Ständestaat « erhoben, weil die Handlungsfähigkeit und Entscheidungsfreiheit des Staats vom Konsens gesellschaftlicher Gruppen abhängig gemacht werde ${ }^{58}$. Abgesehen von der schon oben aufgezeigten Mystifizierung »des Staats« verkennt dieser Einwand den wesentlichen Unterschied zwischen dem mittelalterlichen »Ständestaat« und dem Klassenstaat der kapitalistischen Gesellschaft, in dem jeweils charakteristischen gesellschaftlich-geschichtlichen Gesamtzusammenhang ${ }^{60}$. Die ständische Ordnung beruht auf der Haus- und Herrengewalt als einheitlicher, zugleich privater und öffentlicher Gewalt, die keine Trennung in öffentliche und private Herrschaft kannte. Okonomische und rechtliche Stellung stimmten insoweit überein, als jedes vollberechtigte Glied - nicht Privatperson - ein Stück "Staatsgewalt" besaß, das er jedoch nur über den Stand vermittelt in den "politischen " Entscheidungsprozeß einfließen lassen konnte. Das ständische System war demnach gekennzeichnet durch dauernde Herrschaftsprivilegien im Rahmen von vielschichtigen vertikalen Abhängigkeitsverhältnissen, woraus vielfache Konsenspflichten der "zentralen Landesgewalt* resultierten. Demgegenüber hat die privatrechtliche Verfügungsgewalt über das kapitalistisch fungierende Eigentum die ökonomischen Schranken des einzelnen Haushalts durchbrochen und ist sunpolitisch. geworden, d.h. sie hat die private, wirtschaftliche Sphäre und die öffentliche Gewalt auseinander treten lassen. Mit der Unterscheidung zwischen sozialer Ungleichheit und staatsbürgerlicher Gleichheit ist ein einheitliches politisches Entscheidungs- und Steuerungszentrum entstanden, das über die gesamte Gesellschaft reicht und infolge der (zunehmenden) Interdependenz aller sozialen Bereiche den gesamtgesellschaftlichen Zusammenhang vermittelt durch seine politischen Entscheidungen. Jedoch wirkt diese politische Entscheidungsinstanz des Staats nicht losgelöst von, nicht über den gesellschaftlichen Prozessen, die vielmehr ihre - für alle geltenden - Entscheidungen bedingen. Wie die privatwirtschaftlich fundierte Vorherrschaft der kapitalistischen Klasse alle anderen gesellschaftlichen Bereiche ergreift, wird auch der Staat zum offiziellen Repräsentanten der Einheit der Gesellschaft insofern, als er tendenziell der Staat der vorherrschenden Klasse ist. Die Klassen der kapitalistischen Gesellschaft sind nicht eingeordnete Teile eines gegliederten Ganzen, sondern wollen - jede für sich *Alles« sein, d. h. die Vorherrschaft über Gesellschaft und Staat erlangen. Das

58 Vgl. dazu Däubler, S. 205 ff.

50 So Quaritsch, S. 36, 45, 51; auch จ. Münch, ZBR I970, S. 374,376

60 Vgl. zum Folgenden O. Brunner, Land und Herrschaft, 4. Aufl. 1959, S. 238, 253, 337 ff., 402 ; Heller, Staatslehre, I934, S. II 4 ff.; Habermas, Strukturwandel der Offentlichkeit, I962, S. I7, 27 ff., 40 f.; U. Preuß, S. I3I ff., 184 ff.; Däubler, S. 193 ff. 
gilt nicht nur für das Bürgertum und die kapitalistischen Interessen, sondern ebenso für die Arbeitnehmer: Die sozialstaatliche »Privilegierung « des Streikrechts zerstört daher nicht die einheitliche Entscheidungsgewalt des Staats (soweit sie als eine sozial bedingte überhaupt besteht), sondern gesteht den Arbeitnehmern nur das den sozialen Bedingungen adäquate Aktionsmittel zu, damit auch sie entsprechend dem die ganze Gesellschaft einheitlich durchziehenden Klassengegensatz wie die Unternehmerseite auf den staatlichen Entscheidungsprozeß einwirken können. 